\title{
Efficacy and safety of ultrasound-guided serratus anterior plane block with different doses of dexmedetomidine for patients undergoing modified radical mastectomy: A randomized controlled trial
}

Caiping Duan ( $\square$ nmgduancaiping@126.com )

Ordos Central Hospital

Zaijun Hao

Ordos Central Hospital

Junling Yang

Ordos Central Hospital

Fei Song

Ordos Central Hospital

\section{Xiujuan Fan}

Ordos Central Hospital

Ruizhen Li

Ordos Central Hospital

\section{Research Article}

Keywords: dexmedetomidine, modified radical mastectomy, pain management, ropivacaine, serratus anterior plane block, ultrasound

Posted Date: May 6th, 2021

DOI: https://doi.org/10.21203/rs.3.rs-470606/v1

License: (c) (1) This work is licensed under a Creative Commons Attribution 4.0 International License. Read Full License 
7 radical mastectomy: A randomized controlled trial

\section{Efficacy and safety of ultrasound-guided serratus}

\author{
anterior plane block with different doses of
}

dexmedetomidine for patients undergoing modified

Running head: SAPB for patients undergoing MRM

Caiping Duan, M.M.*, Zaijun Hao, M.M., Junling Yang, M.M., Fei Song, M.M., Xiujuan Fan, M.M.

Ruizhen Li, M.M.

Department of Anesthesiology, Ordos Central Hospital, Ordos, Inner Mongolia Autonomous

Region, China

Correspondence: Caiping Duan

Email: nmgduancaiping@126.com

Abbreviations: $M R M=$ modified radical mastectomy, ERAS= enhanced recovery after surgery,

TEB=Thoracic epidural block, INB=intercostal nerve block, PVB=paravertebral block,

USG-SAPB = Ultrasound-guided serratus anterior plane block, Pecs=pectoral nerve,

$\mathrm{DEX}=$ dexmedetomidine, $\mathrm{ASA}=$ American Society of Anesthesiologists, $\mathrm{PCI} A=$ patient controlled

intravenous analgesia, $\mathrm{BMI}=$ body mass index, $\mathrm{APS}=$ acute pain service, $\mathrm{VAS}=$ visual analog 
scale, $\mathrm{BIS}=$ Bispectral index, $\mathrm{PACU}=$ post-anesthesia care unit, $\mathrm{PONV}=$ Postoperative nausea and vomiting, LOS= level of sedation, QoR-40=40-item Quality of Recovery questionnaire, $\mathrm{MAP}=$ Mean arterial pressure, $\mathrm{HR}=$ heart rate, $\mathrm{SD}=$ standard deviation, $\mathrm{IQR}=$ inter-quartile range, TTPB=transversus thoracic muscle plane block, PSI=parasternal intercostal block

Abstract

Background: Ultrasound-guided serratus anterior plane block (USG-SAPB) has been used for pain management of patients undergoing modified radical mastectomy (MRM), but evidence supporting the adjuvant analgesic benefits is limited. We explored the efficacy and safety of preemptive use of different concentrations of dexmedetomidine and ropivacaine in USG-SAPB for patients undergoing MRM. Patients and methods: Ninety-five female patients were randomly allocated to RD1 and RD2 groups. USG-SAPB was performed before anesthesia induction. Consumption of sufentanil, postoperative pain scores, and level of sedation were recorded 1-72 h postoperatively. Intraoperative hemodynamics, PACU length of stay, incidence of moderate-severe pain, one-time puncture success, block procedure time, time to first rescue analgesia, satisfaction scores of patients and surgeons, hospital length of stay, adverse events, the prevalence of chronic pain, and quality of postoperative functional recovery were recorded.

Results: Dynamic VAS was significantly lower at 4, 8, and $12 \mathrm{~h}$ after surgery and sufentanil need was significantly lower at $4,8,12,24$, and $48 \mathrm{~h}$ after surgery in the RD2 group $(\mathrm{P}<0.05)$. The incidence of moderate-severe pain was significantly lower in the RD2 group ( $P<0.05)$. Time to first rescue analgesia was significantly longer in the RD2 group ( $P=0.047)$. Consumption of 
43 propofol, remifentanil, dexmedetomidine, use of vasoactive agents, and PACU length of stay

44 (LOS) were significantly reduced in RD2 patients $(P<0.05)$. There were no significant differences

45 between the two groups with respect to procedural variables or satisfaction scores of patients

46 and surgeons, and/or postoperative complications. The hospital LOS, global QoR-40, and

47 prevalence of chronic pain were comparable. Conclusions: Use of $1 \mu \mathrm{g} / \mathrm{kg}$ dexmedetomidine and ropivacaine in USG-SAPB can provide superior postoperative analgesia for patients undergoing MRM without additional adverse effects, and result in similar quality of postoperative functional recovery and prevalence of chronic pain.

52 Keywords: dexmedetomidine, modified radical mastectomy, pain management, ropivacaine, serratus anterior plane block, ultrasound 


\section{Introduction}

Breast cancer is the most common cancer in women worldwide, accounting for nearly

58

59

60

61

62

one-third of all new cancer cases in the female population. ${ }^{1}$ Modified radical mastectomy (MRM)

is currently the first-line treatment for early, localized breast cancer despite the increasing use of chemotherapy and endocrine therapy before surgery. ${ }^{2} \mathrm{~A}$ previous study has reported that over $35 \%$ of patients experience acute postoperative pain following MRM despite the surgical methods being minimally invasive. ${ }^{3}$ Postoperative acute pain has been recognized as a high risk factor in the development of postmastectomy pain syndrome, having an incidence rate as high as $25-60 \%$, with a consequent impaired quality of their life. ${ }^{4,5}$ Furthermore, one of the most important reasons for such a high incidence of postoperative pain may be due to the limited attention to perioperative pain relief compared with the other cancer surgeries. ${ }^{6}$

Multimodal analgesia, an important component of enhanced recovery after surgery (ERAS), can effectively reduce surgical stress and pain-related complications, leading to early mobilization and shortened hospital stays. ${ }^{7,8}$ Regional anesthesia, one of the most used opioid-sparing strategies, has been frequently preferred as part of multimodal analgesia for its improved analgesia and fewer side effects in recent years. ${ }^{9,}{ }^{10}$ Thoracic epidural block (TEB), intercostal nerve block (INB), paravertebral block (PVB), and local infiltration are common methods of postoperative analgesia after MRM. However, each method has its own advantages and disadvantages. ${ }^{11-14}$ As a result, less invasive multimodal analgesia strategies are being investigated. Thoracic plane blocks have been recently proposed as a novel and rapidly expanding facet of regional anesthesia, and in particular, ultrasound guidance has been 
introduced to improve the success rate and safety of regional nerve block. ${ }^{15,16}$ 2013, has been used for pain control after breast surgery because of its excellent analgesia, reduced invasiveness, simplicity, easy-to-learn technique, and relative safety. ${ }^{17}$ It can block the lateral branches of the intercostal nerves of the T2-T9 spinal nerves by injecting local anesthetic into the plane, either superficial or deep to the serratus anterior muscle. As a result, it can provide analgesia in the chest wall, axillary region, and posterior region of the shoulder. ${ }^{18}$ USG-SAPB has been used in chest drain insertion, reconstructive breast surgery, cosmetic breast surgery, and video-assisted thoracoscopic surgery. ${ }^{19}$ Compared with other thoracic planar blocks such as pectoral nerve (Pecs) I and II blocks, the local anesthetic is injected to a more dorsal region in SAPB. As a result, SAPB may target the thoracic nerves more selectively and anesthetize a greater number of intercostal nerves. ${ }^{20}$ Local anesthetic combined with dexmedetomidine (DEX) has been reported to prolong analgesia in brachial plexus blocks. ${ }^{21}$ However, it is presently unclear if DEX combined with ropivacaine will provide superior postoperative analgesia of the SAPB. The aim of this study was to explore the efficacy and safety of preemptive use of different concentrations of DEX and ropivacaine in USG-SAPB for patients undergoing MRM. 


\section{Methods}

\section{Patients}

97
This trial was designed in accordance with the CONSORT 2010 statement. Ethical approval was obtained from the Institutional Review Boards of Ordos Central Hospital and registered at Chinese Clinical Trial Registry (09/06/2020, ChiCTR2000033685). Written informed consent was obtained from patients before trial participation.

Patients who underwent breast cancer surgery in our hospital between June and September 2020 were recruited. Inclusion criteria were the following: patients with American Society of Anesthesiologists (ASA) I-II, age 45 to 60 years, scheduled for unilateral MRM with axillary lymph node dissection, received patient controlled intravenous analgesia (PCIA) at least for $72 \mathrm{~h}$ after surgery. Exclusion criteria: patients with contraindications to SAPB (ie, anticoagulant treatment or coagulative abnormality, infection at injection site, severe chest wall deformities), known allergies to the drugs used in the study, radiation before surgery, secondary surgery, smoking, or drug abuse, history of motion sickness, peripheral neuropathy (eg, diabetic neuropathy), severe cardiopulmonary disease, renal and liver dysfunction, history of chronic pain, unable to cooperate or communicate, body mass index $(\mathrm{BMI})>30 \mathrm{~kg} / \mathrm{m}^{2}$, refusal to participate in the block procedure.

\section{Randomization and blinding}

Patients were randomly allocated by a computer-generated random number list. The results were concealed in sequentially numbered envelopes that were opened after enrolment of 
patients. All participants were unaware of the group assignment. Nurses in the acute pain service (APS) team educated patients how to use the visual analog scale (VAS) and PCIA pump, prepared the study drugs, and performed postoperative assessments.

\section{SAPB Procedure}

No premedication was given before SAPB. Patients received standard ASA monitoring (ie, noninvasive blood pressure, pulse oximetry, electrocardiography, and temperature) after arriving at the anesthesia preparation room and venous access was established at the contralateral upper limb. Bispectral index (BIS) monitoring electrodes were placed on each side of the patient's forehead according to the manufacturer's instructions. Patients were sedated and received analgesia with a $0.02 \mathrm{mg} / \mathrm{kg}$ midazolam and $1 \mu \mathrm{g} / \mathrm{kg}$ fentanyl was administered after applying nasal cannula oxygen at $2 \mathrm{~L} / \mathrm{min}$. The same anesthesiologist with sufficient training in ultrasound performed the real-time SAPB under USG as described in a previous study. ${ }^{22}$ Briefly, patients were placed in the lateral decubitus position with the operation side up, a high-frequency linear probe was placed over the middle clavicular region in a sagittal plane after routine disinfection. Then, a 22-gauge needle was introduced from caudad to cephalad using plane technology after the subcutaneous tissue, latissimus dorsi, serratus anterior, intercostal muscle, and pleura were identified at the fourth rib in the midaxillary line. After confirming the location of the needle with $2 \mathrm{~mL}$ of saline solution without blood or air in aspiration, $20 \mathrm{~mL} 0.5 \%$ ropivacaine with $5 \mathrm{mg}$ dexamethasone and $0.5 \mu \mathrm{g} / \mathrm{kg}$ or $1 \mu \mathrm{g} / \mathrm{kg}$ DEX was injected in the deep layer of serratus anterior plane (between the serratus anterior muscle and external intercostal muscles) for about 10 seconds. We defined a successful block as the loss of cold sensation in more than 
two dermatomes $30 \mathrm{~min}$ after the block and then were transferred to operating room. Otherwise, the procedure was considered as a failure of block.

\section{Anesthesia}

Anesthesia was induced by lidocaine $1.5 \mathrm{mg} / \mathrm{kg}$, dexamethasone $0.1 \mathrm{mg} / \mathrm{kg}$, fentanyl 2-3 $\mu \mathrm{g} / \mathrm{kg}$, propofol 1-2 mg/kg, and cisatracurium $0.1 \mathrm{mg} / \mathrm{kg}$. A laryngeal mask was positioned to control the airway during surgery. DEX $0.2-0.7 \mu \mathrm{g} / \mathrm{kg} / \mathrm{h}$, propofol 3-6 $\mu \mathrm{g} / \mathrm{mL}$, remifentanil $0.05-0.2 \mu \mathrm{g} / \mathrm{kg} / \mathrm{min}$ were titrated to maintain BIS at $50-60$ and hemodynamics within $20 \%$ of baseline. Cisatracurium $0.05 \mathrm{mg} / \mathrm{kg}$ was given at the discretion of anesthetists. Tropisetron $5 \mathrm{mg}$ and ketorolac $30 \mathrm{mg}$ were intravenously administered about $30 \mathrm{~min}$ before the end of the surgery.

Neuromuscular block was reversed by neostigmine $0.02 \mathrm{mg} / \mathrm{kg}$ and atropine $0.01 \mathrm{mg} / \mathrm{kg}$ if necessary. A volume of $10 \mathrm{~mL} 2 \%$ lidocaine was administered by subcutaneous infiltration in the parasternal and subclavicular areas at the end of the operation for postoperative analgesia by the surgeon according to previous study. ${ }^{23}$ The laryngeal mask was removed after patients responded promptly to the verbal command and patients were shifted to the post-anesthesia care unit (PACU). MRM was performed using the same technique by the same surgical team according to previous study. ${ }^{24}$

\section{Postoperative Pain Management}

Patients received the same postoperative analgesia protocol in both groups. At the end of surgery, PCIA was started with $0.8 \mu \mathrm{g} / \mathrm{mL}$ sufentanil. The bolus volume was $2 \mathrm{~mL}$, background dose was $1 \mathrm{~mL} / \mathrm{h}$, locked time was $5 \mathrm{~min}$, and the 1 -h limit was $12 \mathrm{~mL}$. Intravenous flurbiprofen 
axetil injection $50 \mathrm{mg}$ was administered if VAS $>3$. Postoperative nausea and vomiting (PONV) was treated with $5 \mathrm{mg}$ tropisetron.

\section{Data collection}

The primary outcome was consumption of sufentanil 72 hours after operation. Secondary outcomes included postoperative pain scores: VAS $0 \mathrm{~cm}=$ no pain, $10 \mathrm{~cm}=$ worst imaginable pain) and level of sedation (LOS, 6-point scale: $0=$ =alert; $1=$ mildly drowsy; 2=moderately drowsy, easily arousable; 3=very drowsy, arousable; $4=$ difficult to arouse; and 5=unarousable), which were recorded at $1,2,4,8,12,24,48$ and $72 \mathrm{~h}$ postoperatively, intraoperative hemodynamics, PACU length of stay, PONV (5-point scale: $0=$ no nausea, $1=$ mild nausea, $2=$ severe nausea, $3=$ one episode of vomiting, 4=vomiting more than once), incidence of moderate-severe pain (dynamic VAS $>3$ ), one-time puncture success, block procedure time, time to first rescue analgesia, satisfaction scores of patients and surgeons (5-point scale: 1=extremely unsatisfied; $5=$ extremely satisfied), hospital length of stay, adverse events and the prevalence of chronic pain.

The quality of postoperative functional recovery (40-item Quality of Recovery questionnaire (QoR-40): 40 to 200) was assessed the day before surgery, the first day, on discharge, and three months after surgery.

Mean arterial pressure (MAP) and heart rate (HR) were recorded at the following time points: arrival at the operating room (T0), before SAPB procedure (T1), after SAPB Procedure (T2), before anesthesia induction (T3), after anesthesia induction (T4), before incision (T5), immediately after skin incision (T6), and extubation (T7). Hypotension was defined as MAP decreased by $>20 \%$ compared with baseline and treated with $40 \mu \mathrm{g}$ phenylephrine or $6 \mathrm{mg}$ 
177 ephedrine. Bradycardia was defined as $\mathrm{HR}<60$ beats/min or decrease $>20 \%$ compared with 178 baseline and treated with $0.2 \mathrm{mg}$ atropine.

\section{Statistical Analysis}

180

The sample size was calculated on the basis of a $15 \%$ reduction in the cumulative amount of sufentanil $72 \mathrm{~h}$ after the surgery in our preliminary trial $(85.92 \pm 24.08 \mu \mathrm{g}$ in the RD1 group). For a study power of $80 \%$ ( $\alpha=0.05, \beta=0.2$ ), 42 participants were required each group according to PASS 11.0 (NCSS Statistical Software, Kaysville, UT, USA). Assuming a dropout rate of $15 \%$, the final sample size was determined to be 49 patients in each group.

The Kolmogorov-Smirnov test was used to assess distribution of variables. Homogeneity of variance was determined using Levene's tests. Quantitative data were expressed as the mean \pm standard deviation (SD) or median and inter-quartile range (IQR). Differences between groups were compared using repeated-measures analysis of variance with the Bonferroni correction for normally distributed data. For nonnormally distributed data, groups were compared with the Mann-Whitney $\mathrm{U}$ test. Categorical data were expressed as number, frequency, or percentage, and analyzed using chi-squared tests or Fisher's exact tests. Time between surgery completion and first request of rescue analgesics was plotted as Kaplan-Meier survival curves and compared using the Log rank test. $P<0.05$ was considered statistically significant. Statistical analysis was performed with SPSS for Windows Version 22.0 (SPSS Inc. Chicago, IL, USA). 


\section{Results}

\section{Baseline Characteristics}

A total of 201 patients who underwent MRM in our hospital between June and September

2020 were recruited using the CONSORT diagram (Fig 1). Overall, 103 patients were excluded

for the following reasons: ASA of 15 patients $>$ II, age of 27 patients $<45$ or $>60$ years, 7 patients

with contraindications to SAPB, 3 patients radiation before surgery, 2 patients with the secondary

with peripheral neuropathy, 8 patients with history of chronic pain, 5 patients unable to cooperate or communicate, 11 patients with $\mathrm{BMI}>30 \mathrm{~kg} / \mathrm{m}^{2}$, and 14 patients who refused to participate in

the block procedure. Finally, 98 patients were included and divided into two equal groups: Group

RD1 (20 mL 0.5\% ropivacaine with $5 \mathrm{mg}$ dexamethasone and $0.5 \mu \mathrm{g} / \mathrm{kg}$ DEX in SAPB) and

Group RD2 (20 mL 0.5\% ropivacaine with $5 \mathrm{mg}$ dexamethasone and $1 \mu \mathrm{g} / \mathrm{kg}$ DEX in SAPB). In

addition, 3 patients were excluded because of the failure of the SAPB procedure (2 patients from

the RD1 group and 1 patient from the RD2 group). There were no significant differences between the two groups with respect to demographic data (Table 1).

\section{Intraoperative variables}


216

217

218

219

220

221

222

223

consumption of cisatracurium, one-time puncture success, or block procedure time $(P>0.05$,

Table 2). The number of patients using vasoactive agents was significantly reduced in the RD2 group during surgery $(P<0.05$, Table 2$)$. Additionally, PACU length of stay was significantly shorter in the RD2 group ( $P<0.01$, Table 2$)$. Although the total dermatomal spread was comparable between the two groups (4 [range, 3-5] vs. 4 [range, 3-5] segments, $P=0.487$ ), more patients in the RD2 group achieved T1and T2 dermatomal spread compared with the RD1 group $(P<0.01$, Table 3$)$.

\section{Postoperative variables}

Compared with the RD1 group, only dynamic VAS was significantly lower in the RD2 group at 4,8 , and $12 \mathrm{~h}$ after surgery $(P<0.05$, Fig 3$)$. Consumption of sufentanil was significantly lower in the RD2 group at 4, 8, 12, 24, and $48 \mathrm{~h}$ after surgery than in the RD1 group ( $P<0.05$, Fig 4$)$. The incidence of moderate-severe pain was significantly lower in the RD2 group $(P<0.05$, Fig 5).

Time to first rescue analgesia was significantly longer in the RD2 group ( $P=0.047$, Fig 6$)$.

There was no difference between the two groups with respect to the level of sedation, or in satisfaction scores of patients and surgeons $(P>0.05$, Table 4$)$.

The most common postoperative complication was PONV, which was more frequent in the RD1 group. However, the difference was not significant $(21.28 \%$ vs. $16.67 \%, P=0.253$, Table 5).

There were no cardiovascular, respiratory, or block-related complications in either group. The hospital length of stay, global QoR-40, and prevalence of chronic pain 3 months after surgery were comparable between the two groups $(P>0.05$, Table 5$)$. 


\section{Discussion}

The findings of this study indicate that USG-SAPB with $1 \mu \mathrm{g} / \mathrm{kg}$ dexmedetomidine could

provide superior postoperative analgesia for patients undergoing MRM. Moreover, there were no significant differences between the two groups with respect to one-time puncture success, block procedure time, the total dermatomal spread, satisfaction scores of patients and surgeons, postoperative complications, the hospital length of stay, global QoR-40, or the prevalence of chronic pain 3 months after surgery.

MRM can be performed under general anesthesia, regional anesthesia, or regional anesthesia combined with general anesthesia. The sources of pain after MRM include incision of surgery, surgical drain, and neuromuscular injury. ${ }^{25}$ It has been reported that this acute pain may transform into chronic pain at a rate of $25-60 \% .{ }^{5}$ As a result, it is very important to control postoperative acute pain effectively. A previous study has demonstrated that the high consumption of opioids may lead to greater pain sensitization through activation of $\mathrm{N}$-methyl-D-aspartate pronociceptive systems, increase post-operative opioid use, and the risk of developing sensory disturbances following surgery. ${ }^{26}$ As a result, regional anesthesia has gained popularity for its opioid-sparing strategies. TEB and PVB are recommended as standard approaches for analgesia as they can reduce respiratory complications, the incidence of postoperative chronic pain, stress-induced immunosuppression, and the systemic PVB, there are still performed with technically difficulty, are time consuming, have high failure 
rates, and complications because of the deep injection site. Furthermore, these methods demands normal coagulation patterns, advanced surgical skills, and a longer learning curve. ${ }^{27}$

Thoracic plane blocks have been proposed as they can offer excellent analgesia and reduce pulmonary complications, stress responses, inflammation, and neurohumoral modulation. ${ }^{28} \mathrm{~A}$ previous study has reported that Pecs I block anesthetizes the medial and lateral pectoral nerves (C8-1) and the anterior cutaneous branches if the local anesthetic penetrates the external intercostals muscles. ${ }^{29}$ Pecs II block is performed to obtain blockade of the upper intercostals nerves; however, it has been avoided due to concerns regarding the disruption of the surgical tissue within the axilla. ${ }^{30}$ Though Pecs blocks could reduce rest and dynamic pain scores and morphine equivalent consumption at 2,12 , and 24 postoperative hours, and required more than one injection. ${ }^{31}$ A previous study reported that Pecs II block may be more effective for short-term postoperative analgesia than SABP during breast cancer surgery. However, this study was a retrospective study and MRM only account for less than $10 \%$ of the surgical procedures. ${ }^{32}$ USG-SAPB is a new approach proposed to provide analgesia to the hemithorax. A previous study has reported that the analgesic effect derived from SAPB is influenced by many factors such as the position of the patient, volume, concentration, the physicochemical characters of the local anesthetic, local tissue conditions, the site and rapidity of injection and whether an adjuvant was used or not, among which the volume maybe the critical factor impacting on the extent of injectate spread..$^{33}$ Compared with other long-acting local anesthetics, such as bupivacaine and levobupivacaine, and ropivacaine has been widely used in regional anesthesia for its larger maximum dosage and lower systemic toxicity and neurotoxicity. ${ }^{34}$ Although Kunigo et al reported 
that SAPB with $40 \mathrm{~mL}$ of $0.375 \%$ ropivacaine diffuses to a greater extent than $20 \mathrm{~mL}$, there was no significant difference between the two groups with respect to the time to first need of analgesic rescue and impact on posterior spread. Furthermore, the clinical analgesic effect cannot necessarily be reflected by cadaveric results evaluating the direct staining of individual nerve branches. As a result, $20 \mathrm{~mL}$ ropivacaine could be safer and would avoid local anesthetic systemic toxicity. ${ }^{20,35}$ A previous study has also reported the comparison of $0.5 \%$ and $0.75 \%$ ropivacaine and showed there was no significant difference in postoperative analgesia, but both were superior to $0.375 \%$ ropivacaine. This indicated that an increased concentration of ropivacaine may be a better approach to improve the analgesic efficacy of SAPB and prolong the duration of pain relief. ${ }^{36}$ Wong et al reported that high concentrations of ropivacaine may lead to respiratory complications. ${ }^{37}$ As a result, we adopted SAPB using $0.5 \%$ ropivacaine for preemptive analgesia in this study. Several reports have shown that low concentrations of ropivacaine can be detected in the blood, which might result in sedation by suppressing the function of the sodium channel in the central nervous system. ${ }^{38,39}$ As a result, we recorded the level of sedation at $72 \mathrm{~h}$ after surgery, although the results were similar between the two groups. The deep block did not disrupt the surgical tissue planes and spared the block of the long thoracic nerve, which would occur in a superficial block, and thus preserved scapula function. ${ }^{40}$ Moreover, the deep block could avoid the need for the injection of local anesthetic into the plane during lymph node dissection or axillary clearance and may promote greater caudad local anesthetic spread. ${ }^{41}$ Thus, we adopted the technique of deep block of SAPB given the higher risk of pneumothorax. ${ }^{42}$ We defined a successful block as the loss of cold sensation in more than two 
dermatomes before surgery instead of after surgery in this study to reduce the recall bias. We used SAPB as the preemptive analgesia before surgery, which might be more effective in reducing hyperalgesia, allodynia, central sensitization, and provides a better risk-benefit ratio. ${ }^{43}$ In addition, DEX probably produces peripheral analgesic effects by inhibiting the transmission of nerve signals through the $\mathrm{A} \delta$ and $\mathrm{C}$-fibers and stimulates the release of enkephalin-like substances in the peripheral regions, although the specific analgesic pathway involved is still unclear. ${ }^{44} \mathrm{~A}$ previous study also reported that corticosteroids, as an adjuvant therapy, can significantly prolong analgesia, decrease postoperative pain while the level of glycemia was only slightly increased on the first postoperative day. ${ }^{45}$ As a result, the postoperative analgesia time of SAPB in our study was longer than that of previous studies. Consistent with the results of a previous study, we also recorded rebound pain $24 \mathrm{~h}$ after surgery. The reason may be due to the higher number of younger female patients recruited in this study who experienced more intense pain after the analgesic effects of SAPB disappeared and an acute state of opioid-induced tolerance and hyperalgesia. ${ }^{46}$

In our study, the time to first rescue analgesia was significantly longer and the rescue analgesia requirement was significantly higher in the RD2 group, which had achieved superior postoperative analgesia. However, there was no difference between the two groups with respect to satisfaction scores of patients and surgeons. Though $10 \mathrm{~mL}$ of $2 \%$ lidocaine was administered by subcutaneous infiltration into the parasternal and subclavicular areas at the end of the operation, most postoperative rescue analgesia requirements were attributed to an incomplete analgesia in the internal mammary area. USG-transversus thoracic muscle plane block (TTPB) 
or parasternal intercostal block (PSI) was recently introduced to provide analgesia in the internal mammary area, which cannot be completely blocked by SAPB ${ }^{47,48}$

Consistent with the results of a previous study, we did not record any complications associated with SABP. The explanation may be that the targeted point of SAPB is superficial and we also adopted real-time USG-SAPB to prevent misplacement of the needle tip in this trial. ${ }^{40}$ Fewer patients in our study experienced PONV than in a previous study, and the reason may be due to treatment with a prophylactic antiemetic before the completion of surgery and the lower consumption of opioids. ${ }^{36}$ In addition, another previous study reported that DEX also reduced the incidence of PONV by reducing the movement of the stomach and intestines, by inhibiting glandular secretion, and by reducing opioid dosage.$^{29}$ Inconsistent with the results of a previous study, we did not record any differences between the two groups with respect to chronic pain, although the consumption of opioids was lower in the RD2 group. This finding can be explained by our recruitment being limited to patients with axillary lymph node dissection, which is an independent risk factor of chronic pain. ${ }^{49}$

Our study had several limitations. First, we did not design a sham group of local anesthetic block with placebo injectate for ethical reasons. Second, we only recruited patients with ASA I-II, although the recruitment of patients with obstructive sleep apnea maybe has been more meaningful. Finally, a larger multicenter prospective randomized double blind study is needed to verify the conclusions of this study. 
339

\section{Conclusion}

Administration of $1 \mu \mathrm{g} / \mathrm{kg}$ dexmedetomidine and ropivacaine in USG-SAPB may provide superior postoperative analgesia for patients undergoing MRM without causing additional adverse effects.

\section{Ethics approval, guidelines and consent to participate}

All procedures performed in studies involving human participants were in accordance with the ethical standards of the institutional and/or national research committee and with the 1964 Helsinki Declaration and its later amendments, or with comparable ethical standards. Ethical approval was obtained from the Institutional Review Boards of Ordos Central Hospital and registered at Chinese Clinical Trial Registry (09/06/2020, ChiCTR2000033685). Written informed consent was obtained from patients before trial participation.

\section{Consent for publication}

Not applicable

\section{Availability of data and materials}

The datasets used and/or analysed during the current study available from the corresponding author on reasonable request 
359 Competing interests

360 Not applicable

361

362 Funding

363 Not applicable

364

365 Authors' contributions

366

Caiping Duan, Zaijun Hao, Junling Yang, and Fei Song conceived and designed the trial;

367

Xiujuan Fan analyzed the data; Caiping Duan, Junling Yang, Fei Song, and Ruizhen Li

368

collected the data; and Caiping Duan, Zaijun Hao and Junling Yang wrote this paper

\section{Acknowledgements}

Not applicable

373 References $7-30$ 

surgery: a prospective study. Journal of Pain. 2006 Sep;7(9): 626-634. surgery: a systematic review and meta-analysis of observational studies. CMAJ. 2016 Oct 4; 188(14):E352-E361.

5. Tian Y, Schofield PE, Gough K, et al. Profile and predictors of long-term morbidity in breast cancer survivors. Ann Surg Oncol. 2013 Oct;20(11):3453-60. block provides more effective perioperative pain relief than pectoral nerves block alone for breast cancer surgery. Br J Anaesth. 2017 Mar 1;118(3):439- 443.

7. Dunkman WJ, Manning MW. Enhanced Recovery After Surgery and Multimodal Strategies for Analgesia. Surg Clin North Am. 2018 Dec;98 (6):1171-1184. fast-tracking to enhanced recovery. J Clin Anesth. 2019 Aug;55:105- 107. 
$0.2 \%$ ropivacaine in combination with ipsilateral brachial plexus block (BPB) for modified radical mastectomy (MRM). J Med Assoc Thai. 2005 Apr;88(4):513-520.

12. Altıparmak B, Korkmaz Toker M, Uysal Al, et al. Evaluation of ultrasound-guided rhomboid intercostal nerve block for postoperative analgesia in breast cancer surgery: a prospective, randomized controlled trial. Reg Anesth Pain Med. 2020 Apr;45(4): 277-282.

13. Li NL, Yu BL, Hung CF. Paravertebral Block Plus Thoracic Wall Block versus

Paravertebral Block Alone for Analgesia of Modified Radical Mastectomy: A Retrospective Cohort Study. PLoS One. 2016 Nov 9;11(11):e0166227.

14. Ferreira Laso L, López Picado A, Antoñanzas Villar F, et al. Cost-effectiveness analysis of levobupivacaine $0.5 \%$, a local anesthetic, infusion in the surgical wound after modified radical mastectomy. Clin Drug Investig. 2015 Sep;35(9):575-582.

15. Galán Gutiérrez JC, Tobera Noval B, Sáenz Abós FJ, et al. Combination of thoracic blocks as a main anesthetic tecnique in modified radical mastectomy for patients with severe respiratory disease. Rev Esp Anestesiol Reanim. 2019 Mar;66(3): 157-162.

16. Grasso A, Orsaria P, Costa F, et al.Ultrasound-guided Interfascial Plane Blocks for Non-anesthesiologists in Breast Cancer Surgery: Functional Outcomes and Benefits. Anticancer Res. 2020 Apr;40(4): 2231-2238.

17. Blanco R, Parras T, McDonnell JG, Prats-Galino A. Serratus plane block: a novel ultrasound-guided thoracic wall nerve block. Anaesthesia. 2013 Nov;68(11):1107- 1113.

18. Mayes J, Davison E, Panahi P, et al. An anatomical evaluation of the serratus anterior plane 

nerves blocks in cardiac surgery, thoracic surgery and trauma: a qualitative systematic review. plane block: a cadaveric study. JA Clin Rep. 2018;4(1):10.

21. Vorobeichik L, Brull R, Abdallah FW. Evidence basis for using perineural dexmedetomidine to enhance the quality of brachial plexus nerve blocks: a systematic review and meta-analysis of randomized controlled trials. Br J Anaesth. 2017 Feb;118 (2):167-181. Anesth Pain Med. 2018 Aug;43(6):644.

24. Othman AH, El-Rahman AM, El Sherif F. Efficacy and Safety of Ketamine Added to Local

433 Anesthetic in Modified Pectoral Block for Management of Postoperative Pain in Patients

434 Undergoing Modified Radical Mastectomy. Pain Physician. Sep-Oct 2016;19(7): 485-494.

435 25. Wang W, Song W, Yang C, et al. Ultrasound-Guided Pectoral Nerve Block I and 436 Serratus-Intercostal Plane Block Alleviate Postoperative Pain in Patients Undergoing Modified 437 Radical Mastectomy. Pain Physician. 2019 Jul;22(4):E315-E323. 
438

439

440

441

442

443

444

445

446

447

448

449

450

451

452

453

454

455

456

457

26. van Gulik L, Ahlers SJ, van de Garde EM, et al. Remifentanil during cardiac surgery is associated with chronic thoracic pain 1 year after sternotomy. Br J Anaesth. 2012 Oct;109(4):

616-622.

27. Luyet C, Eichenberger U, Greif R, et al. Ultrasound-guided paravertebral puncture and placement of catheters in human cadavers: an imaging study. Br J Anaesth. 2009 Apr;102(4): 534-539.

28. Jack JM, McLellan E, Versyck B, et al. The role of serratus anterior plane and pectoral nerves blocks in cardiac surgery, thoracic surgery and trauma: a qualitative systematic review. Anaesthesia. 2020 Oct;75(10): 1372-1385.

29. O'Scanaill P, Keane S, Wall V, et al. Single-shot pectoral plane (PECs I and PECs II) blocks versus continuous local anaesthetic infusion analgesia or both after non-ambulatory breast-cancer surgery: a prospective, randomised, double-blind trial. Br J Anaesth. 2018 Apr;120(4):846-853.

30. Abdallah FW, MacLean D, Madjdpour C, et al. Pectoralis and Serratus Fascial Plane Blocks Each Provide Early Analgesic Benefits Following Ambulatory Breast Cancer Surgery: A Retrospective Propensity-Matched Cohort Study. Anesth Analg. 2017 Jul;125(1): 294-302.

31. Versyck B, van Geffen GJ, Chin KJ. Analgesic efficacy of the Pecs II block: a systematic review and meta-analysis. Anaesthesia. 2019 May;74 (5):663-673.

32. Kubodera K, Fujii T, Akane A, et al. Efficacy of pectoral nerve block type-2 (Pecs II block) versus serratus plane block for postoperative analgesia in breast cancer surgery: a retrospective 

ropivacaine versus bupivacaine for caesarean section: a meta-analysis. Int J Obstet Anesth. 2016 Aug;27:9-16.

35. Kunigo T, Murouchi T, Yamamoto S,et al. Injection volume and anesthetic effect in serratus plane block. Reg Anesth Pain Med. Nov/Dec 2017;42(6):737-740. Res. 2020 Jan 10;13:57-64. concentration $(0.2 \%$ vs $0.1 \%$ ropivacaine $)$ on pulmonary function, and analgesia after ultrasound-guided interscalene brachial plexus block: a randomized controlled study. Pain Med. 2016 Dec;17(12):2397-2403. ultrasound-guided transversus abdominis plane block. Br J Anaesth. 2010 Dec;105(6): 853-856. 
478

479

480

481

482

system toxicity by use of lipid emulsion: effect on total and unbound plasma fractions. J Anesth. 2011 Jun;25(3):442-425.

40. Piracha MM, Thorp SL, Puttanniah V, et al. "A Tale of Two Planes" Deep Versus Superficial Serratus Plane Block for Postmastectomy Pain Syndrome. Reg Anesth Pain Med. Mar/Apr 2017; 42(2):259- 262.

41. Biswas A, Castanov V, Li Z, et al. Serratus Plane Block: A Cadaveric Study to Evaluate Optimal Injectate Spread. Reg Anesth Pain Med. 2018 Nov;43(8):854-858.

42. Desai M, Narayanan MK, Venkataraju A. Pneumothorax following serratus anterior plane block. Anaesth Rep. 2020 Mar 3;8(1):14-16.

43. Ahiskalioglu A, Yayik AM, Demir U, et al. Preemptive Analgesic Efficacy of the Ultrasound-Guided Bilateral Superficial Serratus Plane Block on Postoperative Pain in Breast Reduction Surgery: A Prospective Randomized Controlled Study. Aesthetic Plast Surg. 2020 Feb;44(1): 37-44.

44. Ueda H, Inoue M, Matsumoto T. Protein kinase C-mediated inhibition of mu-opioid receptor internalization and its involvement in the development of acute tolerance to peripheral mu-agonist analgesia. J Neurosci. 2001 May 1;21(9): 2967- 2973.

45. Sir E, Eksert S, Ince ME, et al. A Novel Technique: Ultrasound-Guided Serratus Anterior Plane Block for the Treatment of Posttraumatic Intercostal Neuralgia. Am J Phys Med Rehabil. 2019 Nov;98(11):e132-e135.

46. Kamiya Y, Hasegawa M, Yoshida T, et al. Impact of pectoral nerve block on postoperative 

controlled trial. Eur J Anaesthesiol. 2018 Mar;35(3):215-223. Muscle Plane Block on Postoperative Opioid Consumption After Cardiac Surgery: A Prospective, Anesth. 2016 Oct;30(5):916. post-surgical pain following breast cancer surgery: a systematic review and meta-analysis of observational studies. Br J Anaesth. 2020 Sep;125(3):346-357.

Figure legends

510 Figure 1 Patient enrollment flow diagram.

Figure 2 Intraoperative hemodynamics.

512 Figure 3 Postoperative pain intensity between the two groups. ${ }^{*} \mathrm{P}<0.05$ vs. group RD1.

513 Figure 4 Postoperative sufentanil consumption in both groups. ${ }^{*} P<0.05$ vs. group RD1.

514 Figure 5 The incidence of moderate-severe pain between the two groups. 
Figures

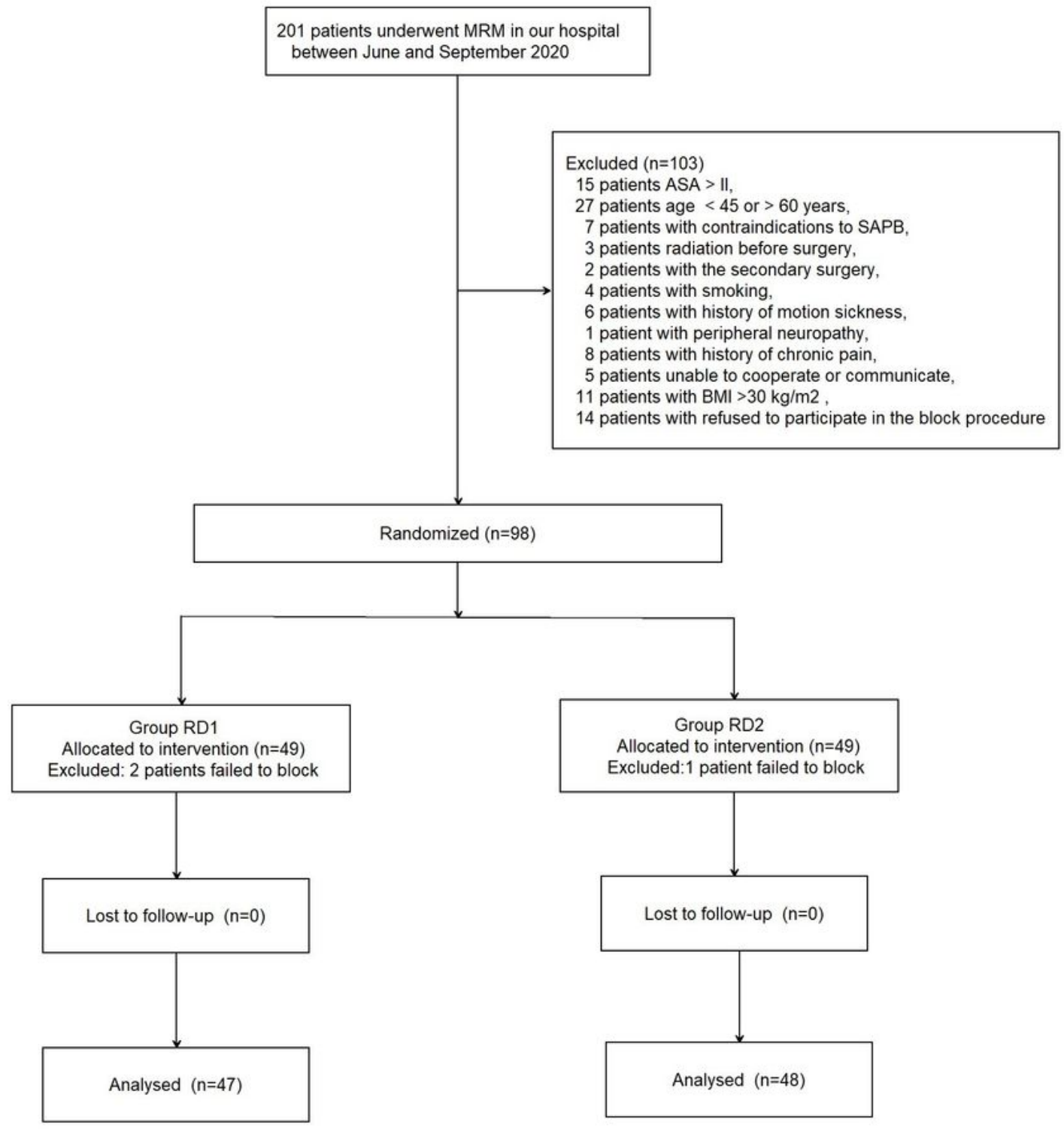

\section{Figure 1}

Patient enrollment flow diagram. 


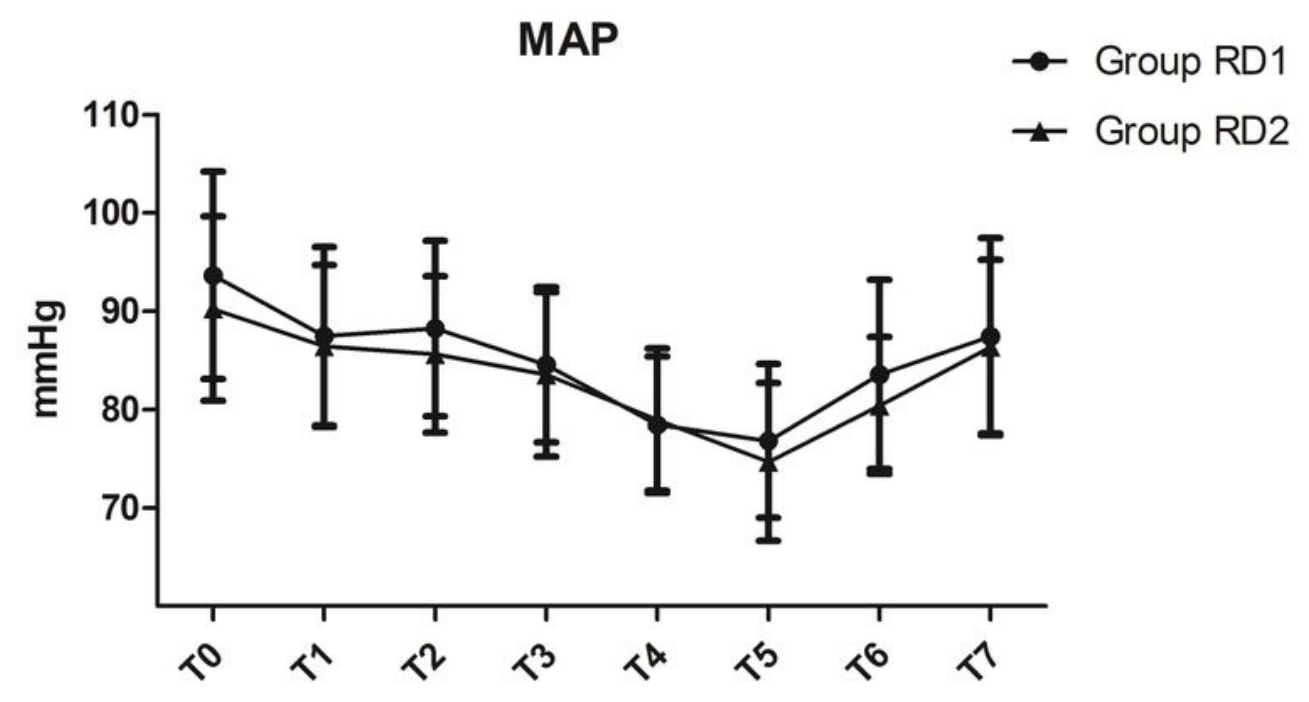

Time

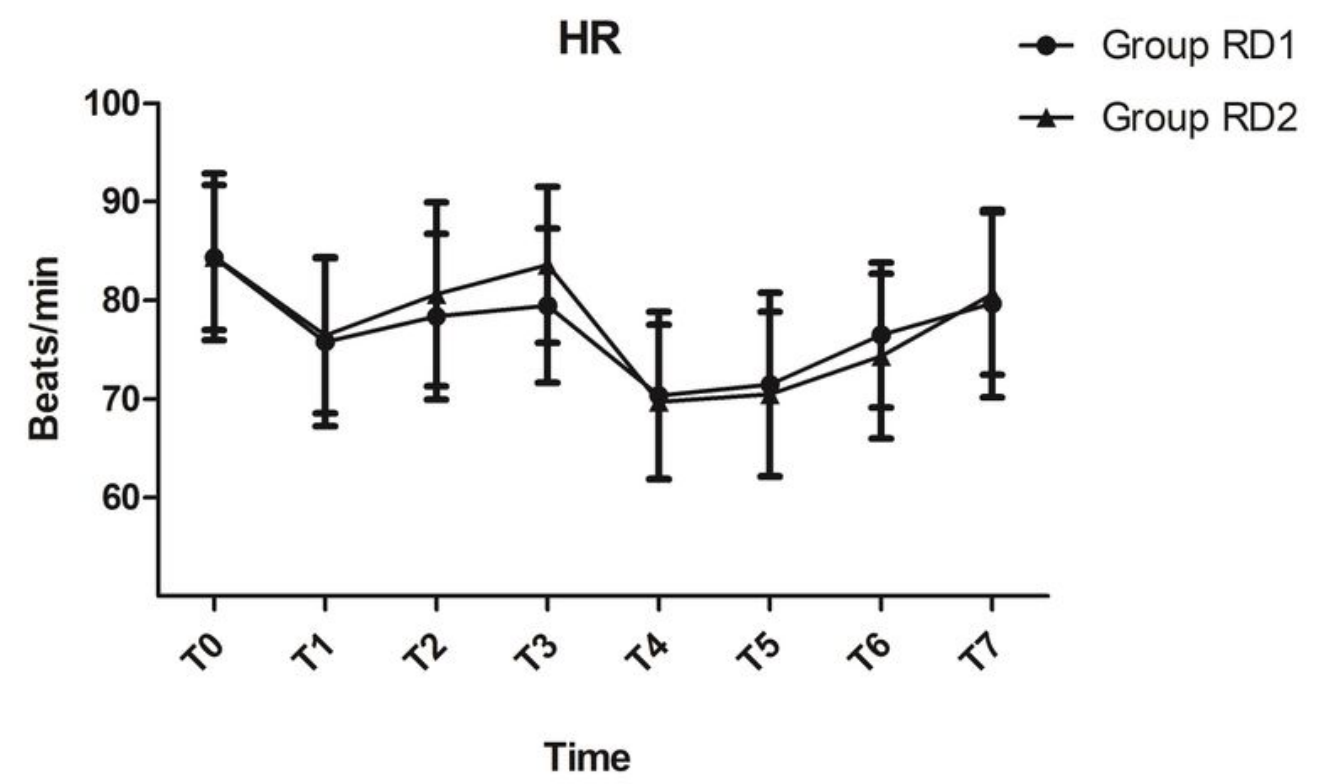

Figure 2

Intraoperative hemodynamics. 


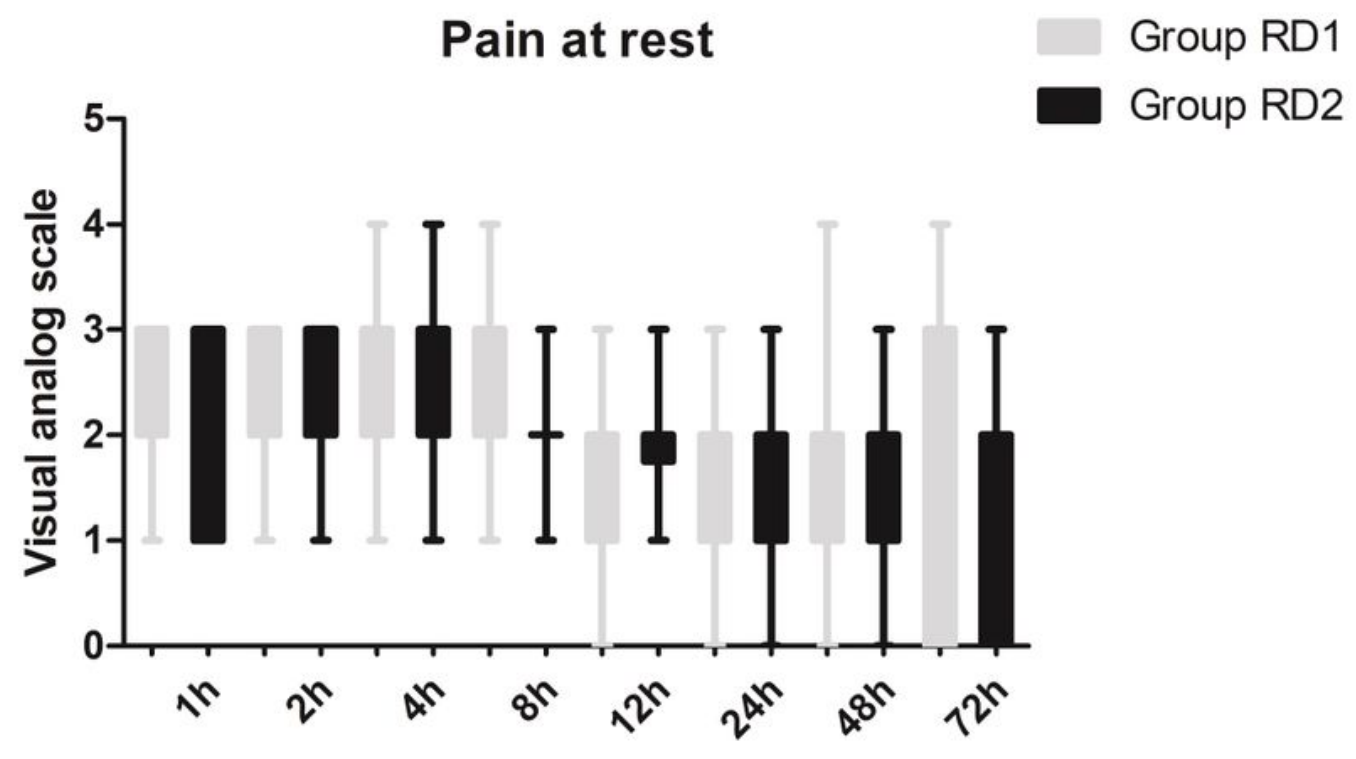

Time

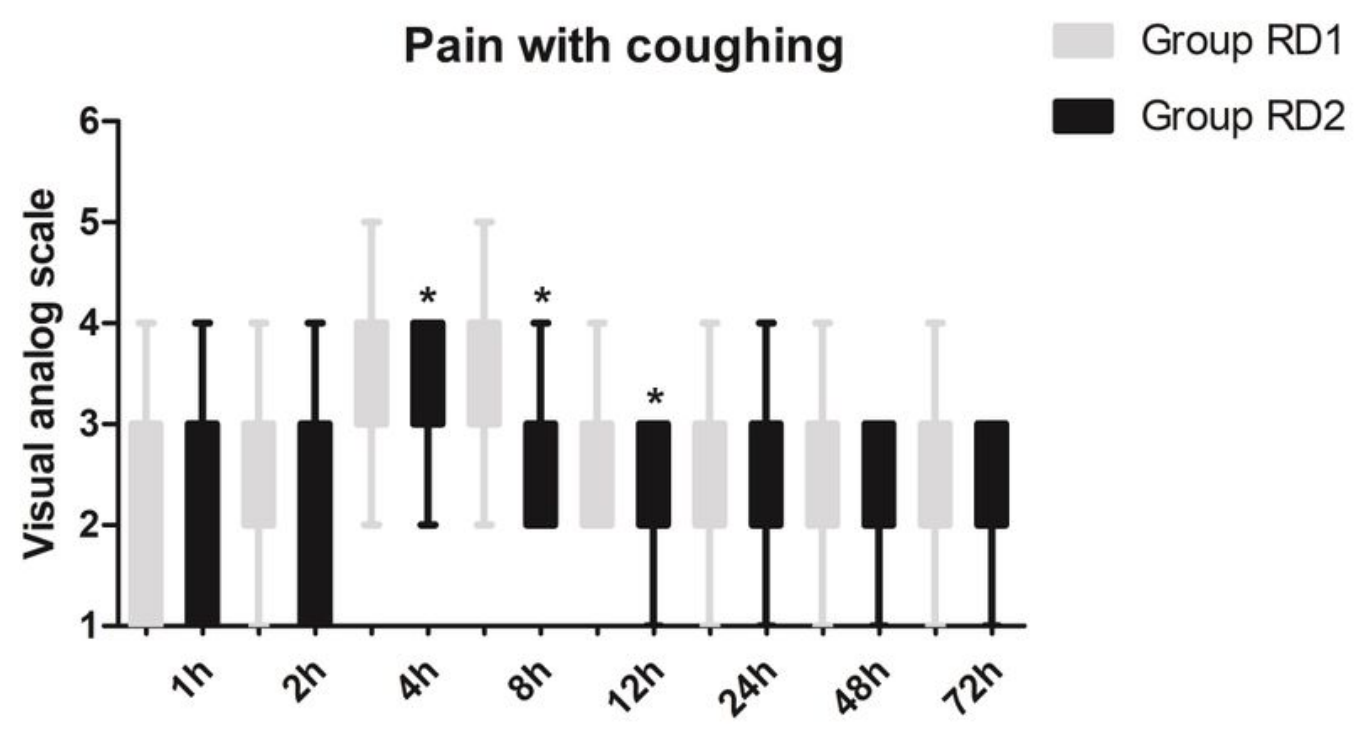

Time

Figure 3

Postoperative pain intensity between the two groups. ${ }^{*} P<0.05$ vs. group RD1. 


\section{Sufentanil}

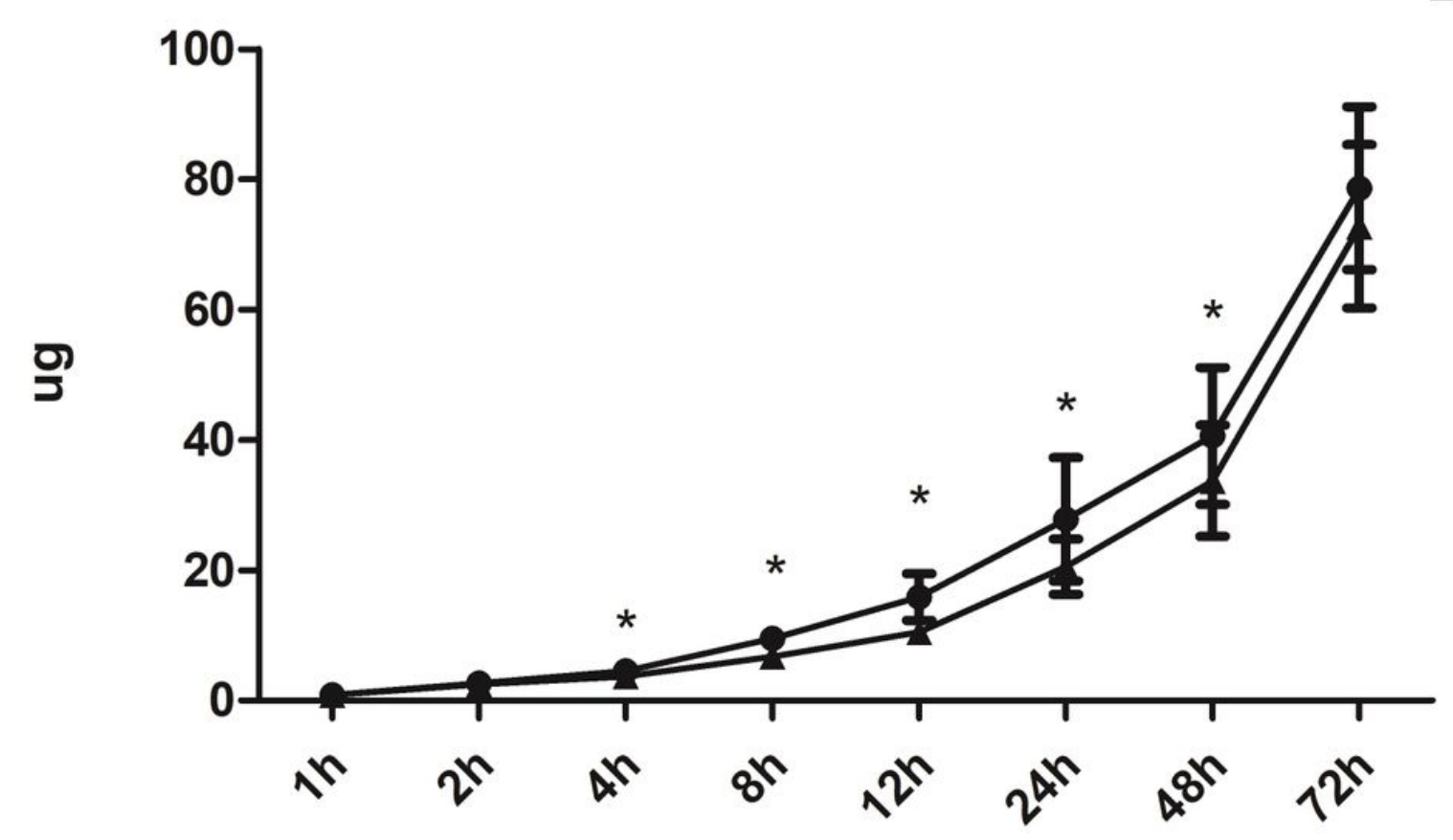

Time $\rightarrow$ Group RD1

$\rightarrow$ Group RD2

Figure 4

Postoperative sufentanil consumption in both groups. ${ }^{\star} \mathrm{P}<0.05$ vs. group RD1.

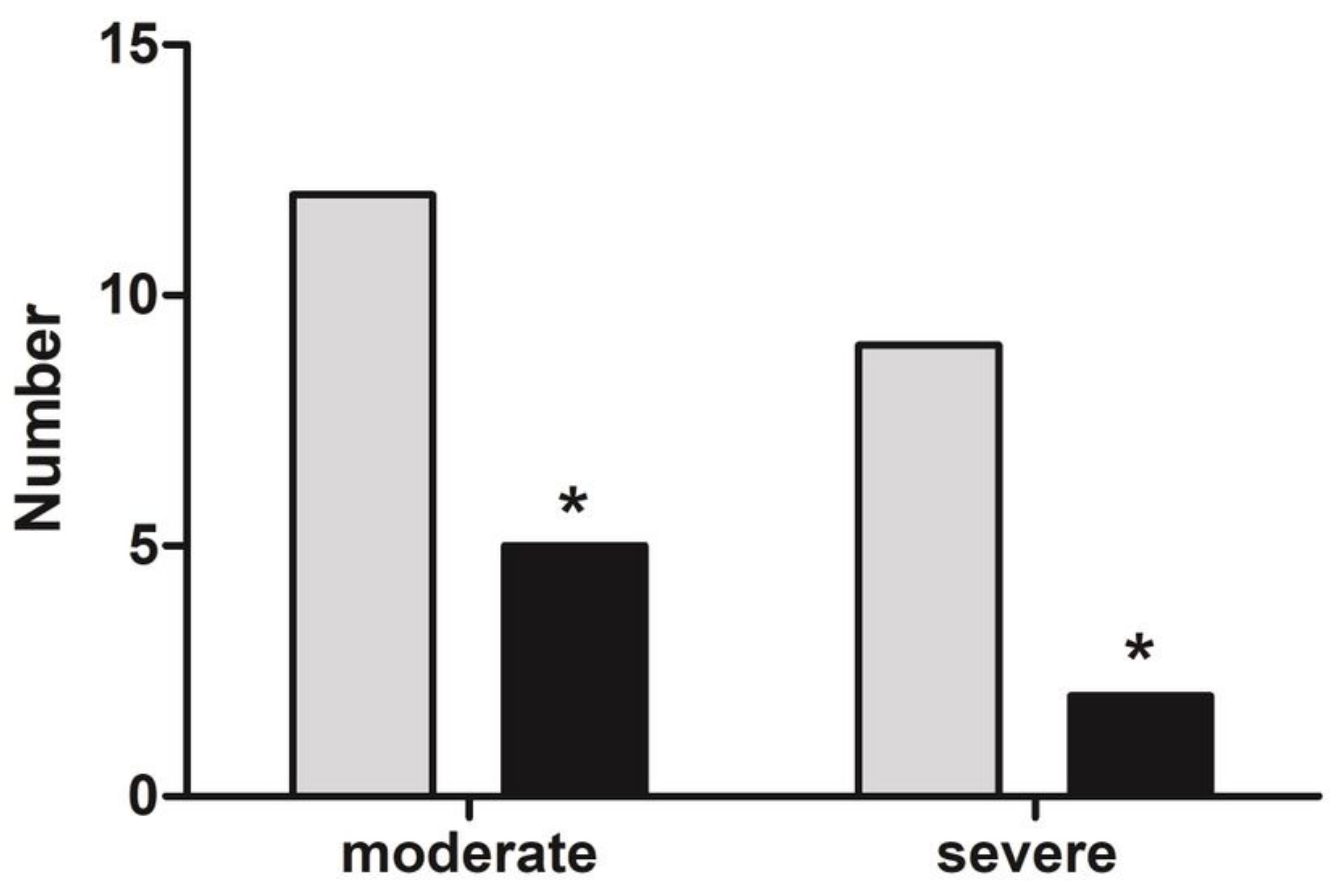

$\square$ Group RD1 - Group RD2 
Figure 5

The incidence of moderate-severe pain between the two groups.

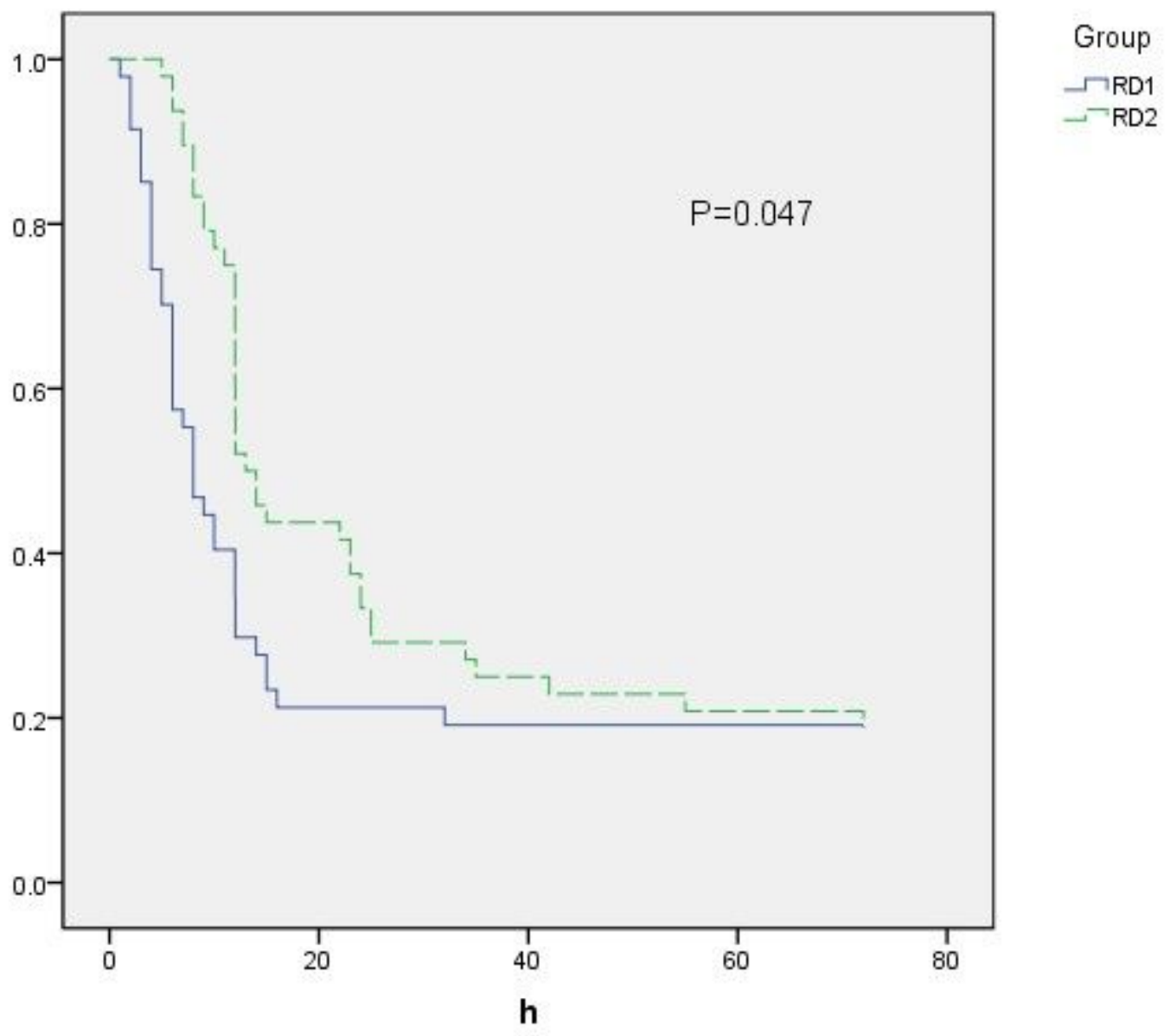

Figure 6

Time to first rescue analgesia. 